\title{
Spatial patterns in mass consumption: The fast food chain network and its street patterns, clusters and impact on street safety
}

\author{
Genevieve Shaun Lin, Kayvan Karimi \\ Space Syntax Laboratory, The Bartlett School of Architecture, UCL. London, UK \\ E-mail: genevieve.lin.16@ucl.ac.uk,k.karimi@ucl.ac.uk
}

\begin{abstract}
Can the fast-food chain network, to some extent, support the sociospatial structure and safety of the street? Is there an urban spatial pattern within the 'Chain Network' and mass consumption? This papers dwells on spatial patterns on mass consumption in the global capitalistic cities of London and Tokyo, through the lens of the fast food chain network. Their symbols (for instance, the Golden Arches of McDonalds) are instantly recognizable both by locals and tourists. McDonalds started off as a hot dog stand in California in the 1940s and rapidly expanded across America in lieu of the mass usage of the automobile and construction of freeways. A foreigner can order easily from a McDonalds menu in Tokyo, without speaking Japanese, because the menu is created in a "global language". Fast food chain stores, such as McDonalds and Starbucks, seem to be sprouting in every street corner, even as much as 3 of the same shop on the same street. You don't have to find them, because they will find you. Rather than casting them aside as complex economic or political factors, the first part of the research focuses on its spatial clustering, and to see if there is an intrinsic spatial relationship with high-choice, or highly integrated streets. How far deep does the network go from the highest choice streets? The second part of the research will see if the clusters of fast-food chain, with their "night economy" would lead to safer and more pleasant street and communities. Fast food chains do indeed play a vital spatial role in our physical communities in the 21st century.
\end{abstract}

Keywords: Space Syntax, Fast Food Chains Network, Spatial Patterns, Mass Consumption, Urban Street Network, Unplanned Buying, McDonaldssystem, poliorcetics, city and territory.

\section{Research Setting}

Fast-food chain networks have been, in most developed cities, relegated to one that is seen as unhealthy, and in some cases signifying a lower class and depicting a crummy, tawdry image. However, they have been observed to serve as "refuge" pit-stops for city-dwellers as well as tourists. For example, a McDonalds in a busy street in Tokyo attracts many foreign visitors because McDonalds has a global symbology that is recognisable to people who don't speak the local language. It is also treated as comfort food because McDonalds has more or less the same menu around the world, with only slight variations. Customers already know what to expect, and usually know what they want without looking at the menu. At the counter, a foreign tourist is also able to order from the menu easily even though it might be written in an unfamiliar language, as the menu is structured in a global language. A businessman or the modern free-lancer is able to use the space comfortably with his laptop and even hold small informal meetings. Young adults congregate at fast-food chains as 
a trendy spot to hang out. WI-FI is provided at most locations. The meals are affordable and self-serviced. One does not need to make extra orders to validate their length of their stay.

These chain-stores produces an interesting architectural spatial narrative in major global cities, though they are usually not appreciated for the extra purposes they serve for the community, urban dwellers and tourists.

The fast food industry kicked off with the McDonald brothers first Hamburger stand in California in the 1940s. It succeeded due to the rise of automobiles and people having the need to stop and have a quick meal when driving, with the least waiting time and costefficient as well. The brothers also, after many iterations, came up with the burger production process, kitchen set-up and configuration to ensure a food making operation, from patty to a burger. Everything was designed for maximum efficiency, smooth process with minimum staff.

McDonalds operates ten of thousands of outlets today, and they make a lot of their profit from real estate business. Today, their famous Golden Arches symbol is known worldwide, no matter which culture one is from. The rise of McDonalds had sparked other fast food chains as well, such as Burger King, KFC, Wendy's etc. Today, in London for example, there is a phenomenal rise in the healthier kind of fast food, such as Wasabi, Pret A Manger, LEON, EAT etc. where one has options of dining in or grabbing a lunchbox to go. This is the reality of modern fast food of the global urban city, where fast food is being sold to people who are health-conscious and aware of nutritional values.

In Death and Life of Great American Cities (1961), Jane Jacobs explains how co-presence, or "eyes upon the street" impacts the nature of street life. Firstly, how shopkeepers were vital to the street because all shopkeepers naturally want safe streets for the good of their shops. Secondly, how people on the streets, even though strangers, through co-presence, keeps an unconscious watchful eye on the streets. This research gives a 21 st century perspective in our global mobile world, where chain stores are a norm. With this chain network theory, and it being a global symbol, how does that relate back to the co-presence that Jane Jacobs talks so passionately about? Does the city 'produce' in some sense the signs of consumption or is it more a vehicle for the 'reproduction' of a consumer-capitalist society? Is its spatiality decodable in these terms? In the recent film, Citizen Jane (2016), Jane Jacobs discusses how the housing projects in the US has failed because of their obscure locations and absence of street life, therefore inciting more potential for crime. A successful street is a well-used street. This shows the importance of vitality of the street and how important pedestrian traffic is.

In Bill Hillier's article Cities as Movement Economies (1998), he suggests that the closest or most accessible places in relation to all others connect centre to periphery, have the highest flows of people and, as a consequence, function as interface between locals and strangers. The main role of space is practical or instrumental. He also states that "if cities are mechanisms for generating contact, then this means that some locations have more potential than others because they have more byproduct and this will depend on the structure of the grid and how they relate to it. Such locations will therefore tend to have higher densities of development, and in turn have a multiplier effect." This 'multiplier' effect is the phenomena that we see on the streets daily, pertaining to the vitality of the street life and flourishing of businesses. This also ties back to the 'co-presence' the Jane Jacobs argues for, for what a successful street is.

This research has significant society impact that firstly, can be defined as the mass consumption network and how consumers are patronising these fast food chain networks as global pit-stops in their daily lives unconsciously. Secondly, understanding the spatial patterns of these mass consumption patterns is vital in addressing issues of micro urban patterns and co-presence, all these would impact how we view and understand urban systems and planning across the global metropolis.

\section{Research Aim and Methodology Research Aim and Questions}

The research aim of the research paper is to 1) Study the intrinsic spatial patterns of the fast- 
food network and analyse the spatial difference of these clusters individually and collectively as an urban network 2) Understand the associations with the city such as retail shop clusters and street crime.

The overarching research curiosity is an attempt to understand spatial patterns of mass consumption in global urban cities, through the lens of the fast food chain network. The curiosity is to also find out if these patterns are different or similar to each other within urban physical space and land-use, and deliberately excluding complex economic or political factors.

1. Is there a relationship between the fast food chain network and spatial street patterns, especially with regards to high-choice street networks?

2. Are there distinguished clusters? How are do they distribute themselves within the cluster?

3. Do fast food chain clusters, in any way, have an effect on street safety? Does it encourage or deter street crime (i.e street violence, robbery, anti-social behaviour etc)

4. Do fast food chain clusters initiate more retail clusters and other restaurants pubs within the same cluster?

My hypothesis is there is a strong relationship between high-choice streets and fast food locations. There is a pattern logic to these clusters. There are distinguished clusters of fast food chains that influences the development of the neighbourhood. With the night economy and co-presence as significant factors, these locations are vital not only to the development of the neighbourhood, but to a certain extent, deter street crime and promote safety of the streets. These fast food locations also go hand in hand with retail and other restaurants/bars to create that effect and plays a part of creating safe neighbourhoods.

\section{Methodology}

There will be two parts to this research. The first part will be to study the intrinsic patterns of the fast food chain locations to reveal their clusters and street network patterns, as well as patterns in their building context. And find out how these clusters work. The second part will be to study relationships between fast food chains and the other events: Crime and Retail In the first part, we will measure the intrinsic spatial patterns of the fast food chain network, and measure them against choice values of each street, based on the Space Syntax theories. We will also see if there is a difference between the different types of fast food group types: Traditional, Modern and Coffee Chains. Following that. We will look out for any spatial patterns in how the clusters are formed using Kernel Density Estimation, Average Nearest Neighbour, to obtain an overall analysis on where and how the clusters are formed. Following that, at the cluster scale, the building and urban context and frontages will be catalogued to spot any common themes.

In the second part, we will study the relationship of the fast food chain network to the society through the lens of street crime rate, as well as compare them with the retail and restaurant land use. Does the chain network affect crime, and does it a have a relationship with the choice value of the street? Does the fast food chain affect the agglomeration of the retail and restaurant location?

Spatial Network Analysis using Space Syntax measures will be used as a baseline to incorporate the above information and factors. This will be elaborated more in the following section.

\section{Measurement and Analysis}

Data Selection Criteria. Based on these following factors, 13 unique fast food chains in London were identified for this research.

Food \& Beverage Physical Store

Chain Store: Minimum 20 outlets in London

Self-service / No waiters taking orders

Quick Service - No or Short waiting time

Takeaway available

Open to everyone

Open all day

Don't have to keep ordering to validate length of stay

Affordable - Generally below $£ 8$ a meal

Casual Environment 


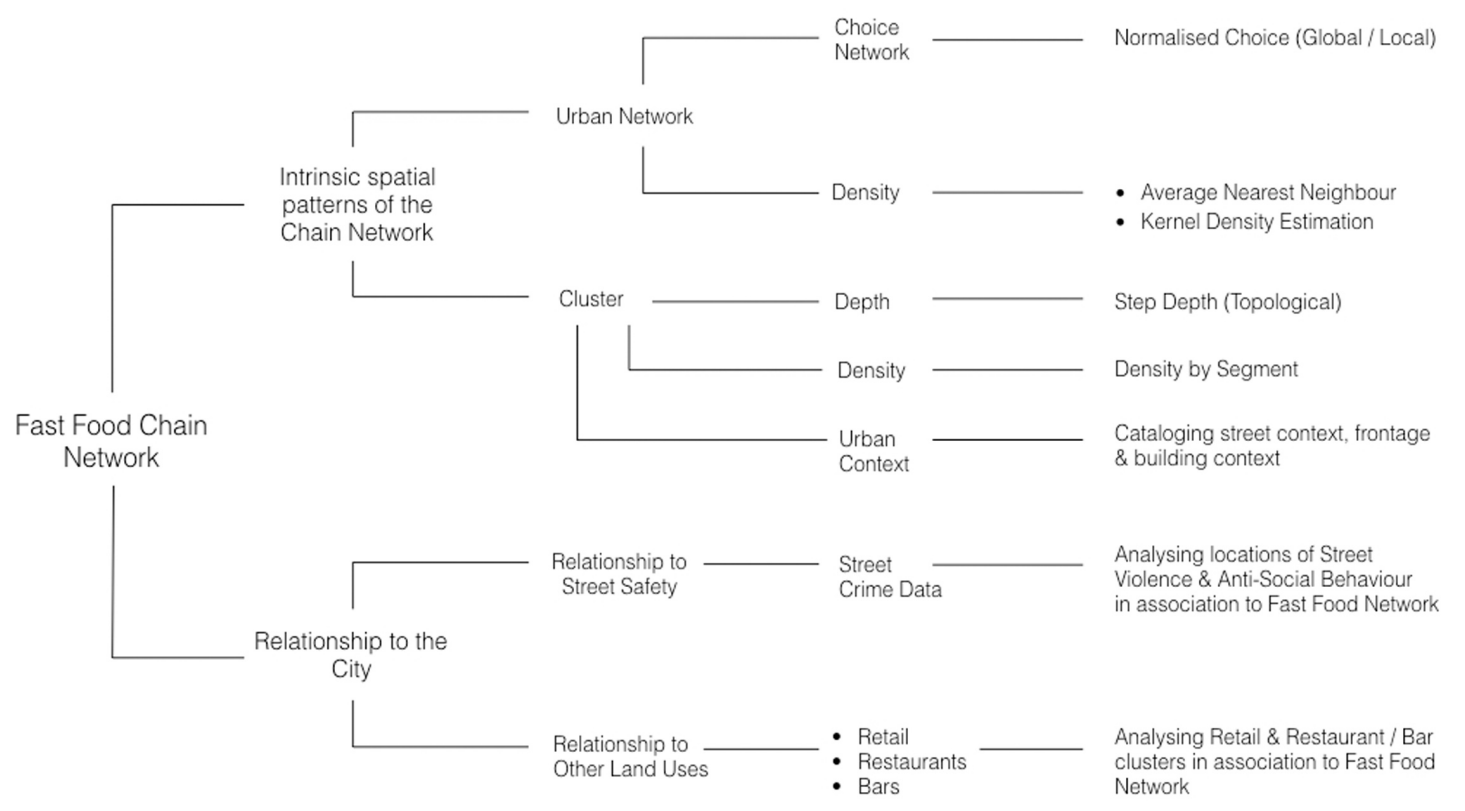

Data Gathering. Based on the results from the Data Selection Criteria, the fast-food locations were gathered through using the Google API. First, geo-data was collected from the chosen 13 fast-food chains in London, from within 30 square kilometres of Trafalgar Square. These geo-data were collected through Google API, using a self-written Python Script, then compiled and edited in a spreadsheet. Following which, they were imported and mapped into geospatial software QGIS, and compared against the results of spatial analysis from Depthmap.

\section{Data Mapping on QGIS Spatial Network Analysis}

Space Syntax spatial segment analysis was conducted on DepthMap such as (Normalised) Integration, (Normalised) Choice at both global and local scales. A 'normalised' calculation allows for a fairer comparison between cities of different sizes, in this case, with Tokyo. Integration values signifies "Tomovement", like a destination point. Choice values signifies "Through-movement", like movement flow, on-route. The result produced was specific to London's urban geometry. The Spatial Network Analysis is independent of real human-movement, economic and political factors. It is based purely on geometry, using applied mathematical formulas, algorithms and selected radiuses.

After that, Step Depth Analysis (Topological) was done to look at the proximity and relationship of the fast food chain clusters from the highest-choice streets. Topological step depth measures proximity by the number of turns away from the origin, rather than by metric or radius.

The aim of this analysis is to apply this base spatial results and compare against the geo-data of the fast food locations and other observations from the entire course of research. The DepthMap analysis serves as baseline for comparison and measurement against empirical information and evidences.

The positions of each fast food store are spatially linked to the street network, with each street having their own unique choice, integration and step depth values. The data is then compiled and assessed to find out the specific spatial patterns, which will be described in the sub-chapters below.

In Figure 3, a comparison is made between the Normalized Global Integration (NAIN Global) of the street networks, and the locations of the fast food chain stores. While there is a relative significant co-relation between these 
two, we will later find out in the study that there is more significant result when compared with Normalized Global Choice (NACH Global). This suggests that the locations and clusters of the fast-food outlets has to do more with pedestrian through-movement and flow, rather than a destination point itself.

\section{Average Nearest Neighbour Analysis}

The Average Nearest Neighbour tool measures the distance between each feature centroid and its nearest neighbour's centroid location. It then averages all these nearest neighbour distances. If the average distance is less than the average for a hypothetical random distribution, the distribution of the features being analysed is considered clustered. If the average distance is greater than a hypothetical random distribution, the features are considered dispersed.

The average nearest neighbour ratio is calculated as the observed average distance divided by the expected average distance (with expected average distance being based on a hypothetical random distribution with the same number of features covering the same total area).

If the index (average nearest neighbour ratio) is less than 1 , the pattern exhibits clustering. If the index is greater than 1, the trend is toward dispersion

\section{Results}

Ratio is 0.3 , which means the fast food chain network in London is highly clustered. This implies that that there is a reason for these clusters, that might pertain to spatial factors. This result tells us that the fast-food chain network, or rather spaces of mass consumption are not random, but these clusters somehow work together through their spatial structure, to contribute to the city's socio-economic mechanism.

\section{Kernel Density Estimation}

Using the Kernel Density Estimation, while adjusting the radius and cell size, the key clusters of the fast food chains are highlighted. These highlighted clusters will be vital when choosing which neighbourhoods should be studied.

\section{Choice Analysis (Normalised, Global)}

Normalised Choice x Cumulative Count Figure 7. (Cumulative Count of Fast Food chains against Normalised Choice values at various scales)

This cumulative count graph shows that, at all scales, from radius $1200 \mathrm{~m}$ to $10000 \mathrm{~m}$ to global (infinite), that $90 \%$ of the locations climbs very fast to a high choice value. Only approximately $10 \%$ of the fast food locations have a lowchoice value on the street network.

What this means is that $90 \%$ of the fast food locations falls on streets with a high-choice values, implying that these fast food locations have a higher chance of people movement opportunity, or through-movement. This is based strictly on the geometry of the street network, using mathematical formulas from the Space Syntax theory of choice analysis and movement.

This might suggest that fast food locations are using the opportunity of high pedestrian movement (foot traffic) to attract people, and does not actually serve as a destination point. This proves the hypothesis true: That you don't have to find the fast food chains because they will find you. One would just "naturally" pass by a fast food outlet, or a series of them.

\section{Step Depth from Highest Choice Streets - Topological}

Topological Step Depth measures proximity of $\mathrm{A}$ to $\mathrm{B}$ by the number of turns, rather than in a metric quantity. Prior to this study, the assumption I had was that most of the fast food chain stores would located on the highest choice streets, while some would perhaps be 1-turning away.

The results of the topological step depth were surprising. Most of the fast food outlets 
were located one step turning away from the highest choice streets, but what is fascinating is there is higher amount of fast food chains located two steps away, more than the highest street themselves.

There are even occurrences of fast food 3 and 4 steps away, though they are few. This led to the next step of doing a density by segment line, to see if it matches the topological step depth results. The results show an intrinsic spatial pattern of fast food small clusters operating on a cross-shaped or L-shaped pattern clusters, where the clusters are located where it can be visibly seen from each other, and cross road junctions are made use by fast-food locations to gain visibility. This is contrary to my initial assumption that all fast food chains merely operate linearly on high streets.

\section{Ongoing Research \\ Relationship of the Chain Network with the City}

Through initial geo-data-mapping studies, it appears that there is a relationship between street crime (anti-social behaviour, street violence) with the positioning of the fast food chain clusters. There seems to be a higher crime rate when these clusters are not located on high-choice streets, but those lower in value, which is uncanny. Also, in comparing in streets within the same neighbourhood, it appears that the streets with fast food clusters does not have crime taking place on the main street where the chains are, but behind on the inner lanes. However, on the adjacent main street where there are no fast food chain outlets, street crime take place on the main street.

The next study will look at retail and restaurant clusters, to see if there is pattern of growth that relates the fast food clusters to other land uses.

\section{Tokyo Study}

In Tokyo, fast food outlets, of those that are similar to London, will be mapped out. Clusters will then be identified and compared against the values of the choice, integration and step depth values. The aim of this chapter of research is to see if where are the commonalities and differences in spatial network across global metropolises.

\section{Summary and Conclusions}

Fast food chain clusters and its network are not located randomly. Rather, over the course of this particular research, they have established a non-discursive spatial network that attempts to be deciphered, qualified and quantified by space syntax measures and theories. As of this pilot stage of the study, the urban network (macro scale) has shown interesting and encouraging results in terms of global and local choice analysis. Approximately $90 \%$ of all fast food chain outlets are located on streets with a highchoice value, at local and global scales. What this actually means is that the fast-food chain network is highly associated with throughmovement (flow) patterns, rather than tomovement (destination points). This discovery alone is a stepping stone towards further investigating individual neighbourhoods and their unique clusters.

At the cluster (neighbourhood) scale, the density by segment analysis was significant in revealing morphological patterns. Unlike what I had speculated before doing this research, fast food chain outlets are not necessarily lined up all in one single axial line. Instead, when the long axial line is studied in individual segments, the fast food outlets breaks up into L-shape pattern clusters. This suggests that these small clusters operate by adjacency, as well as across junctions, to maximize visibility and encourage more pedestrian through-movement. It also reveals that there are occurrences of higherdensity fast food clusters that are located on the high street.

Topological step depth analysis informs us that more of the fast food locations are located one step or two step turning away from the highest choice streets, much more than the highest choice street themselves. Another observation done to see those anomalies led me to find out that this might have to do with the amount of visibility. For example, if the shop is facing an open space such as a park or a fountain square, it can get away with being 
on a lower-choice value street. In my ongoing research, frontage as well as building context will be analysed case by case to discover patterns that might factor in to the locations and sustainability of these clusters.

Finally, the relationship of the fast food chain network and its relationship to the city is equally important as their intrinsic spatial patterns and morphology, Currently, at the Space Syntax Lab, the second part of the research is taking place, regarding the fast food chain network's relationship to crime data as well as patterns of other land use, such as retail, restaurants and bars. This is vital as to find out the unseen connections between fast food and the actual operations of the city. A chapter will be done in Tokyo as well, to compare the relationship between the fast food locations and street network. All these will be completed by September 2017 to be presented at the ISUF conference.

\section{Future Research Directions}

There are a couple of strategies that could potentially evolve from this current research. First, tracking down the growth and sustainability of these fast-fast food chain stores, to determine if stores which has closed down or survived for a long time, has to do with spatial patterns. Since the stores within a single fast food brand sells exactly the same thing with the same food quality, we can tell the effectiveness of the streets by looking at the closure of shops, or the duration of the shop operation, in years.

The overall direction would be to study retail and purchasing patterns in the broader perspective of spatial mass consumption, in association with space syntax and spatial culture. It is a relevant and current phenomenon in today's global world that we can no longer afford to ignore. I would like to clarify that this research does not deliberately benefit mainstream consumption or the retail monopolies, but rather the aim is to seek out underlying spatial patterns in urban networks that could create and re-create successful and safe streets and neighbourhoods for the future.

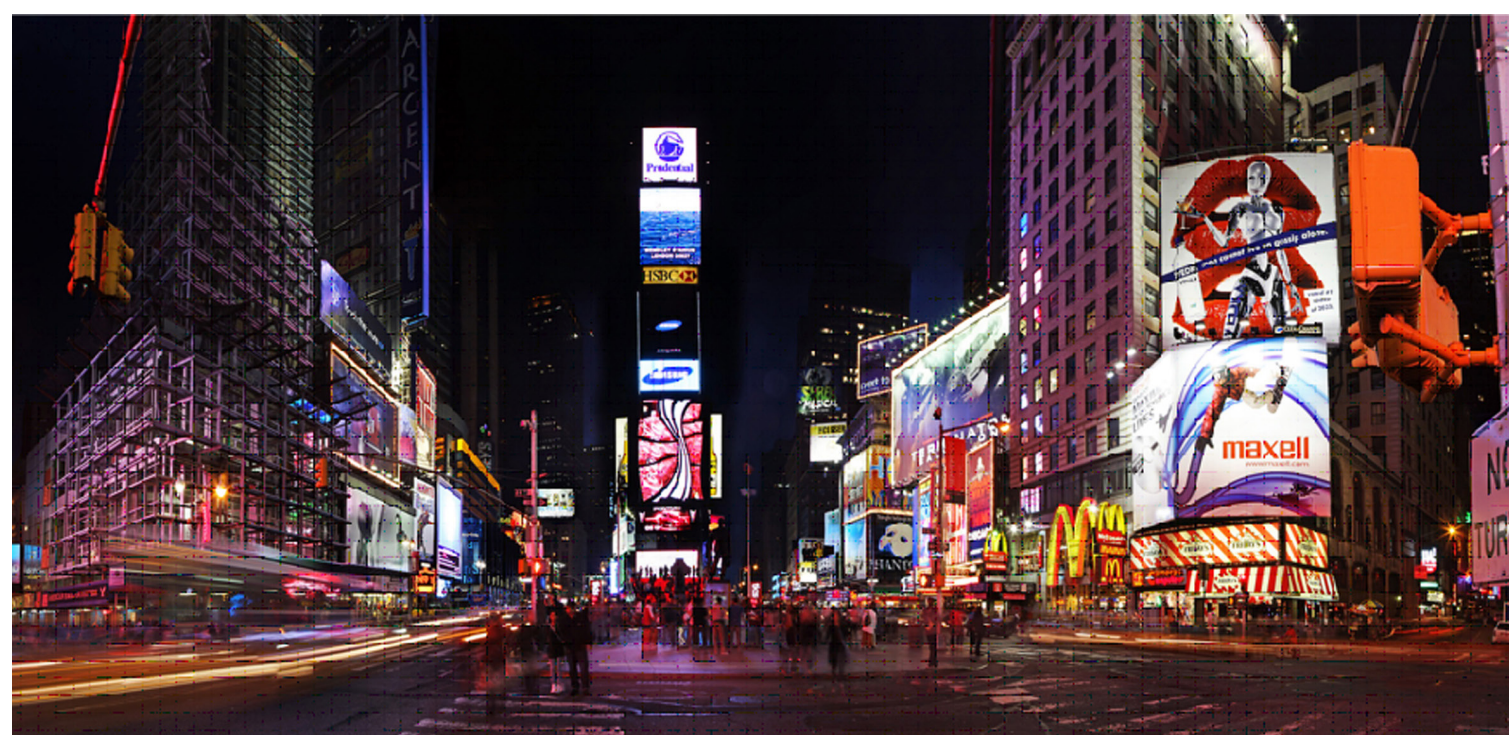

Figure 1. McDonalds signage in Times Square, New York City 


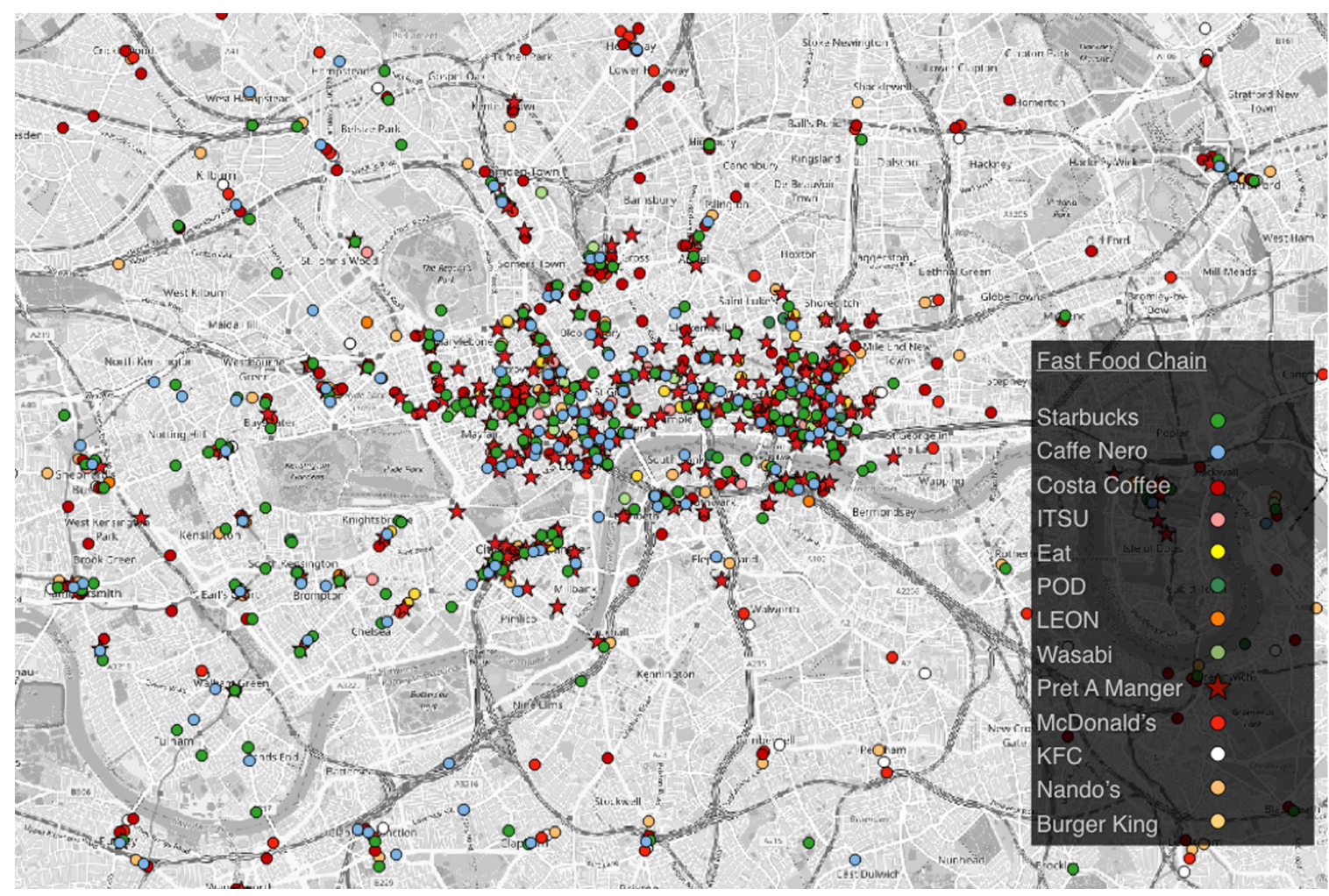

Figure 2. QGIS Data Mapping of 13 fast food chain stores in London

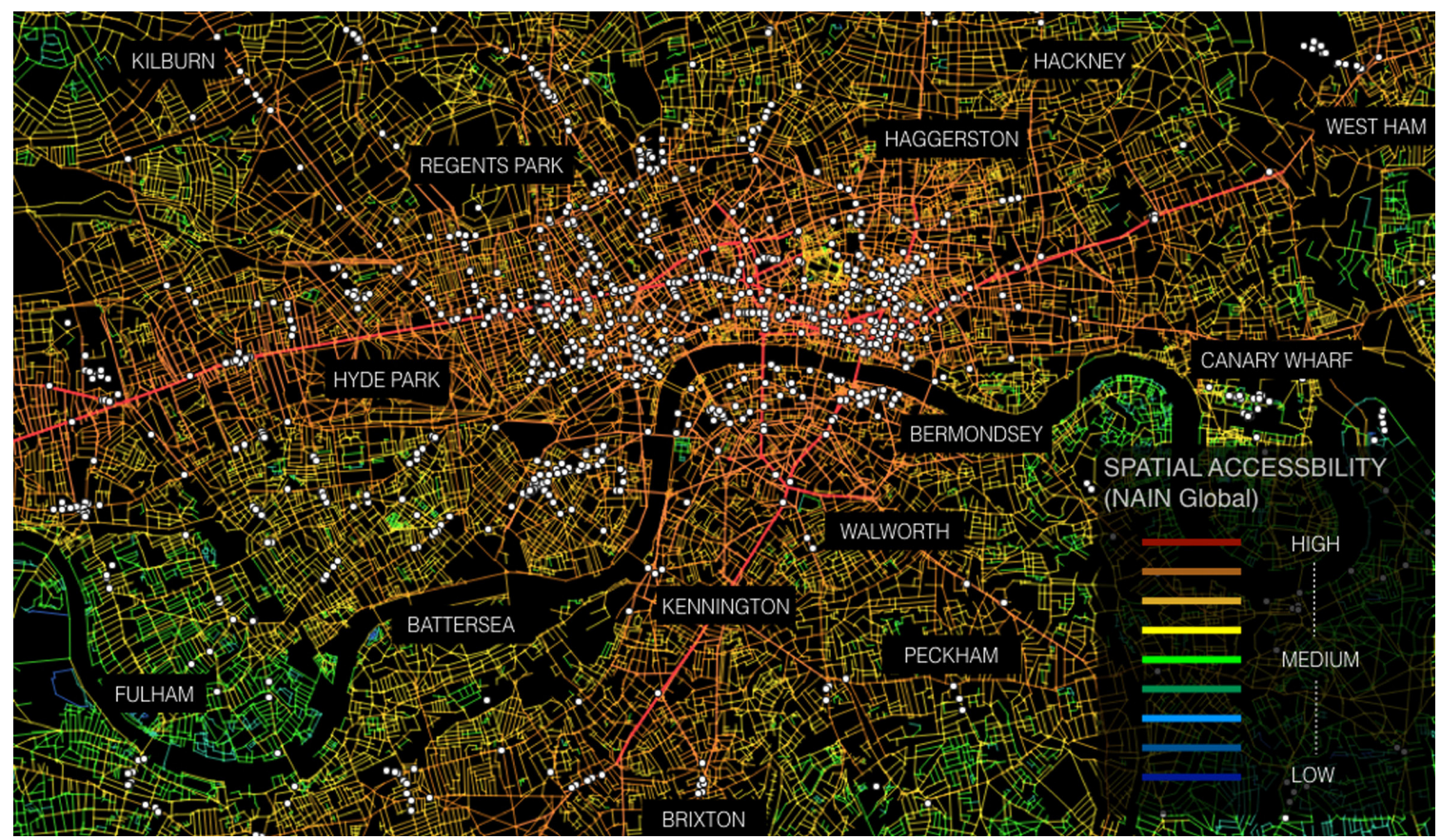

Figure 3. Normalized Global Integration, with all the fast food stores depicted in white circles 

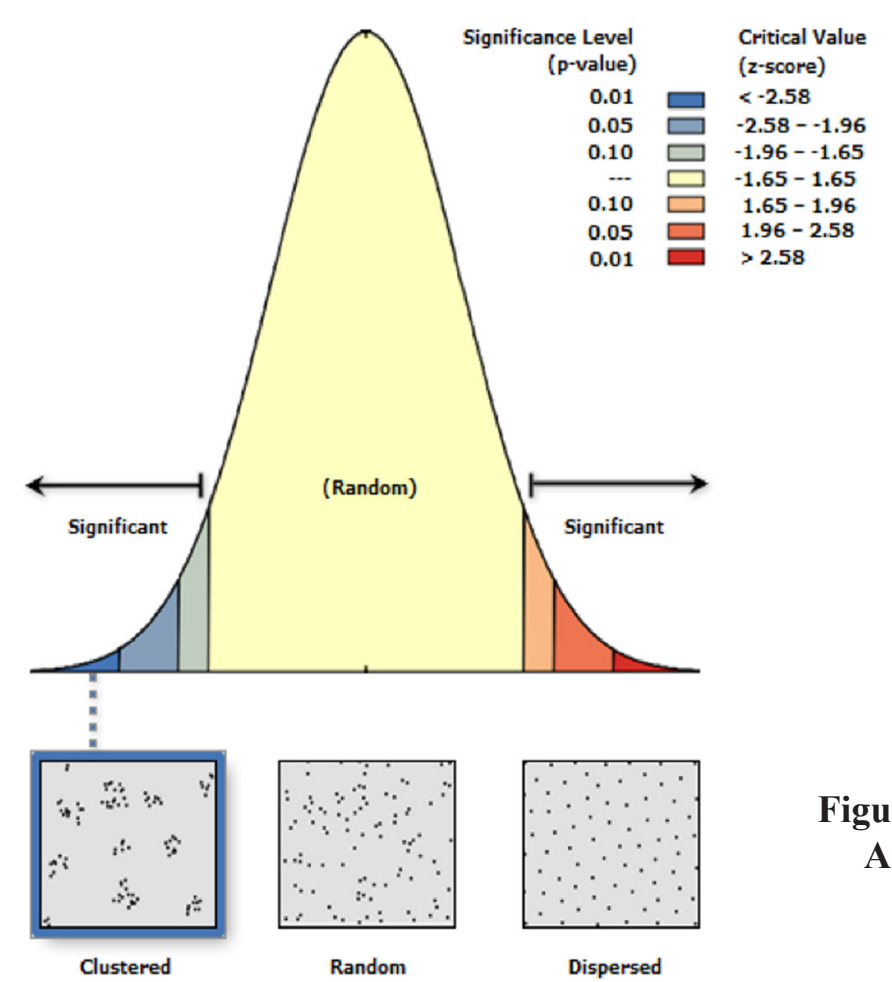

Figure 4. Average Nearest Neighbour Analysis, performed on ARC GIS)

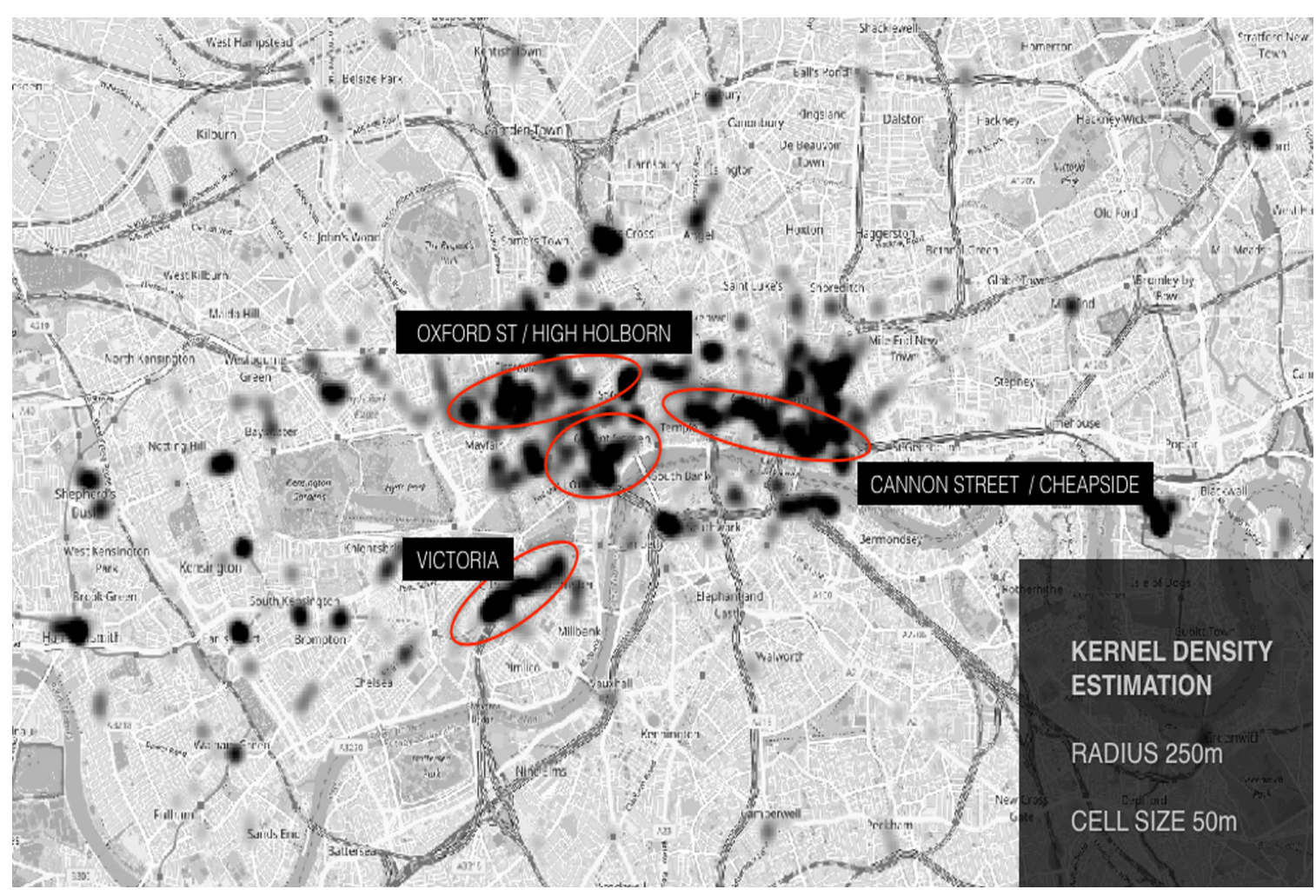

Figure 5. Kernel Density Estimation Analysis, performed on QGIS 


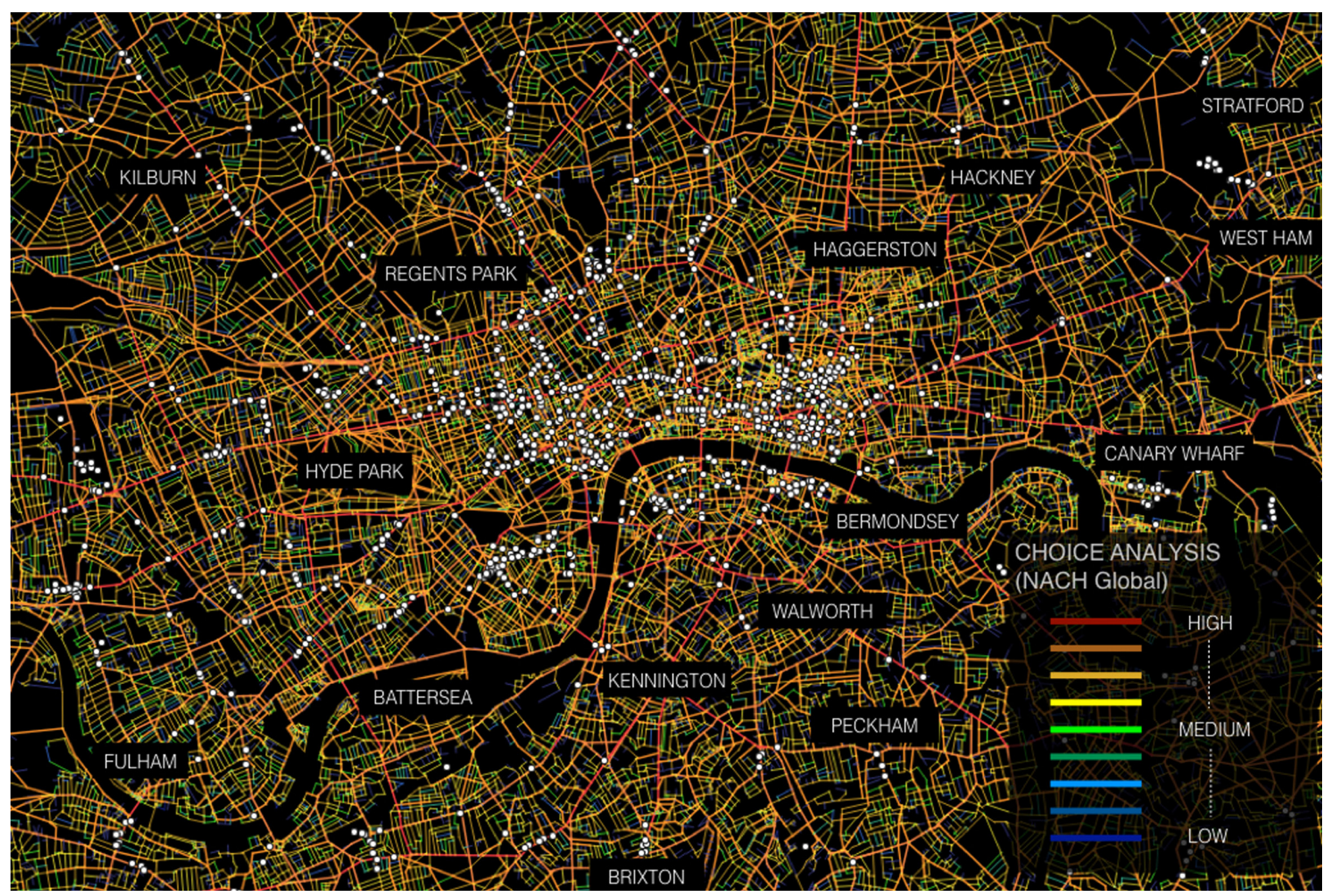

Figure 6. Normalized Global Choice Analysis, with the fast food stores depicted in white circles

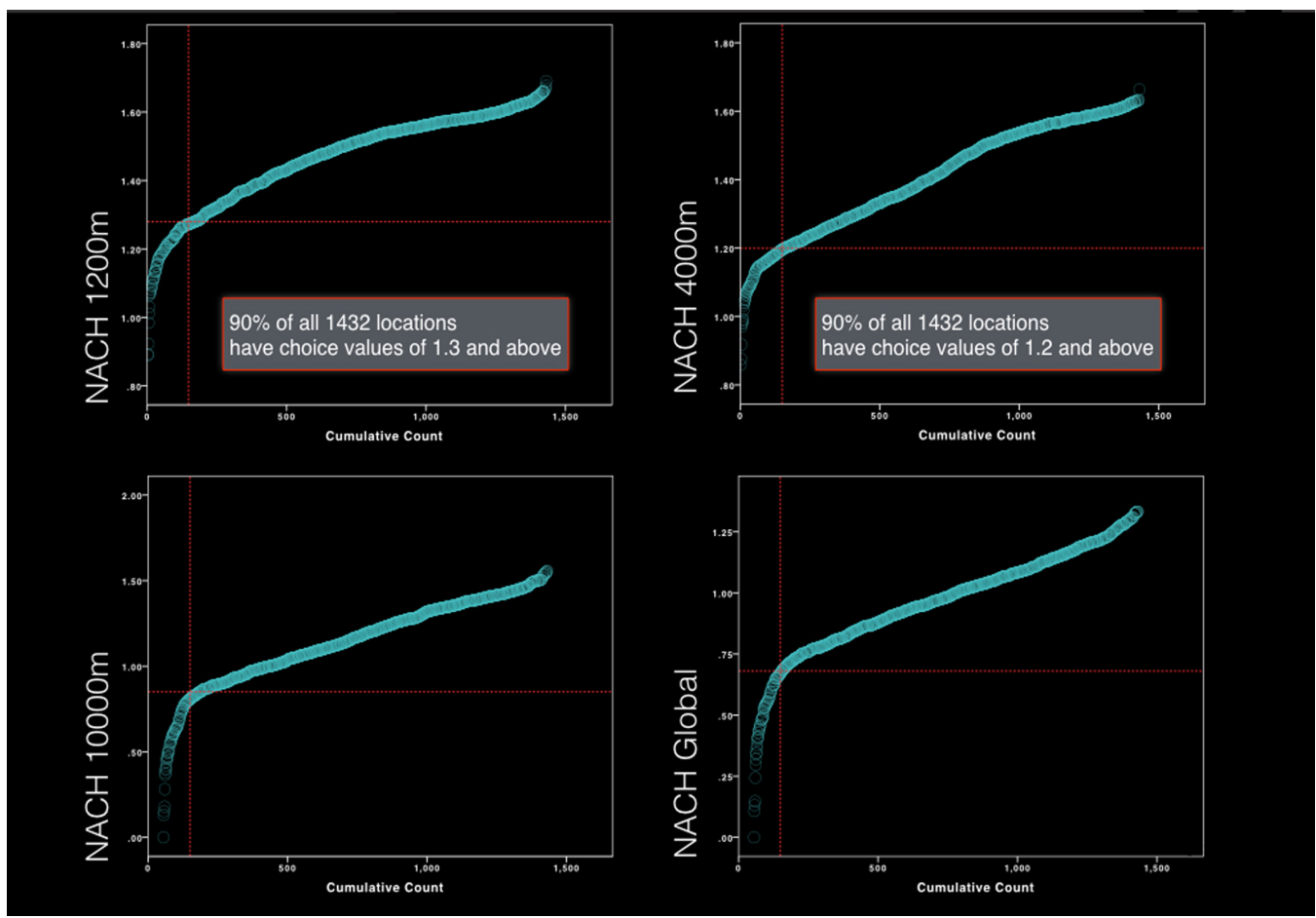

Figure 7. Cumulative Count of Fast Food chains against Normalised Choice values at various scales 


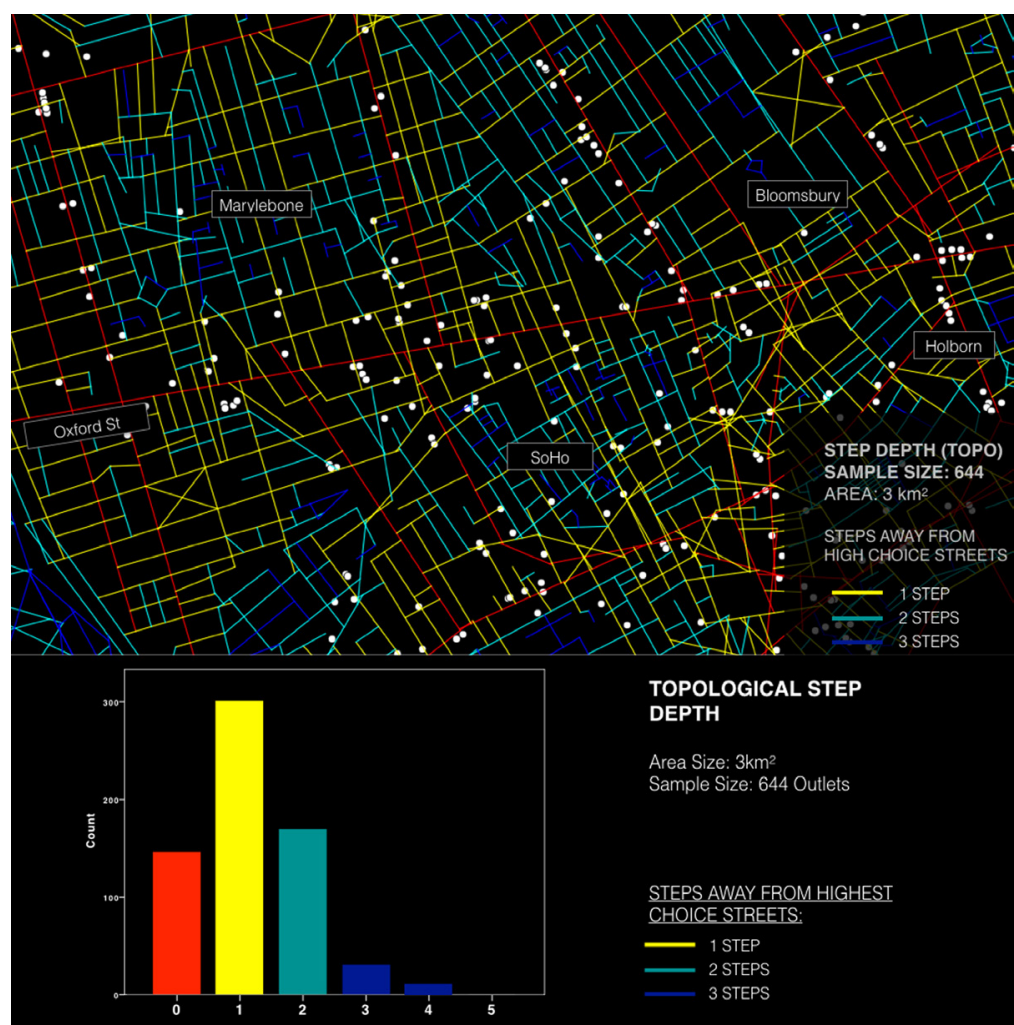

Figure 8. Topological Step Depth Analysis performed on DepthMap
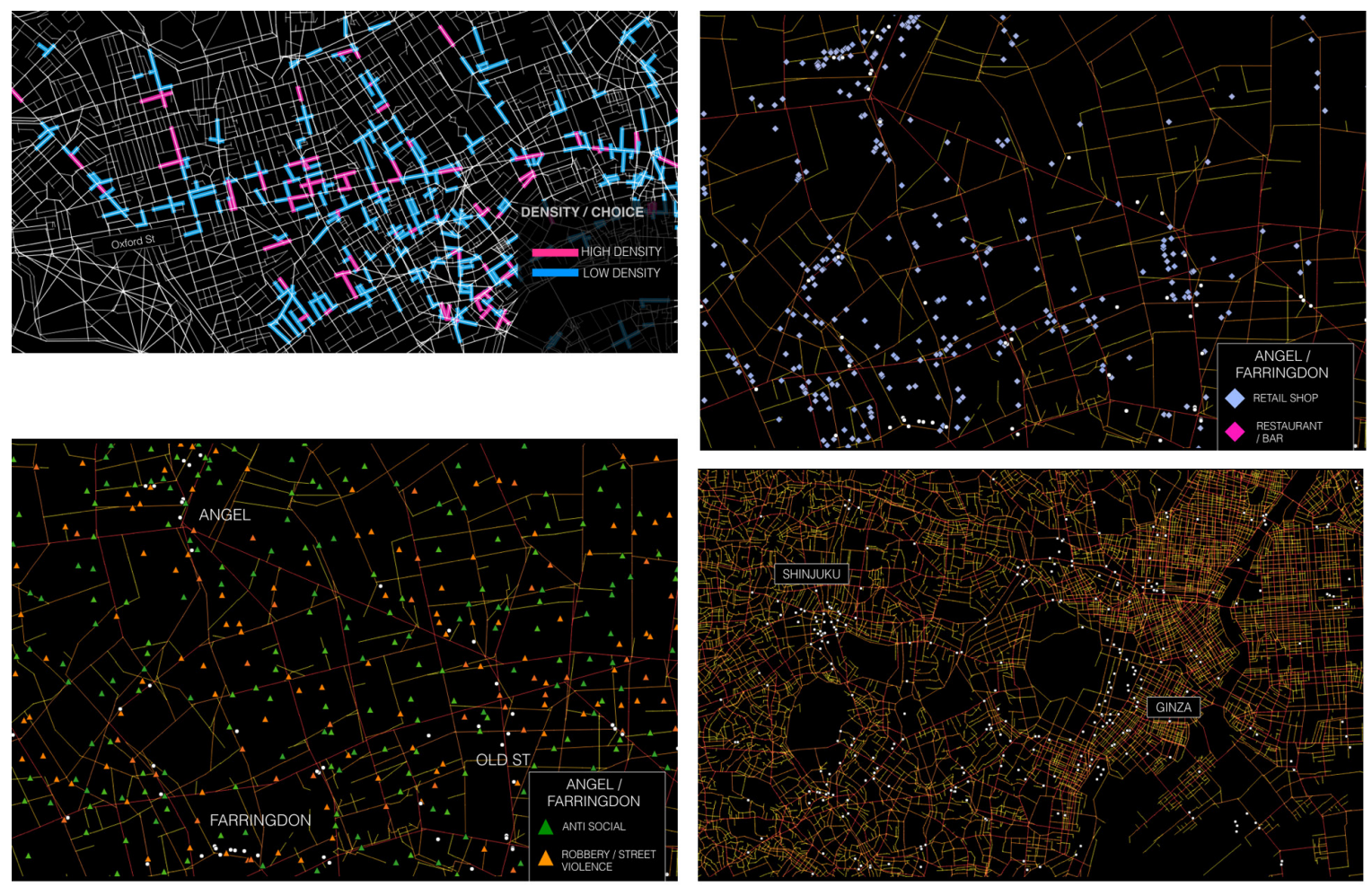

Figure 9. (Above-left)Density of fast food locations by Segment Line in Central London. (Below-left)Mapping of Crime with fast food chain outlets in the City of London. (Above-wright)Mapping of Retail Shops with fast food chain outlets in the City of London. (Below-wright)Ongoing research on the fast food chain network in central Tokyo, Japan 


\section{References}

Boontharm, D. and Radović, D. (2012). Small Tokyo. 1st ed. Tokyo: Flick Studio.

Clammer, J. (2011). Contemporary Urban Japan. 1st ed. New York, NY: John Wiley \& Sons.

Hillier, B. and Hanson, J. (2003). The Social Logic of Space. 1st ed. Cambridge [Cambridgeshire]: Cambridge University Press.

Hillier, B. (1996) Space is the Machine. 1st ed. Cambridge [Cambridgeshire]: Cambridge University Press.

Jacobs, J. (1961). The Death and Life of Great American Cities. New York, Vintage Books.

Lash, S. and Urry, J. (1994). Economies of Signs and Space. 1st ed. London: Sage.

Rook, D. (1987) The Buying Impulse. Journal of Consumer Research14 (2): 189-199. doi: 10.1086/209105

Schlosser, E. (2001). Fast Food Nation. 1st ed. Waterville, Me.: G.K. Hall.

Stern, H. (1962). The Significance of Impulse Buying Today. Journal of Marketing, 26(2), 59-62. doi:10.2307/1248439

Vaughan, L. (2015). Suburban Urbanities. UCL Press.

Venturi, R., Brown, D. and Izenour, S. (1972). Learning from Las Vegas. 1st ed. Cambridge Mass.: MIT Pr.

The Founder. (2016). [film] Hollywood: FilmNation Entertainment.

Citizen Jane: Battle for the City. (2016). [film] New York City: Matt Tyrnauer 